\title{
Approaches for Energy Harvesting and Power Management in Wireless Healthcare Sensor Networks
}

\author{
Anagha Jamthe and Dharma P. Agrawal
}

\begin{abstract}
Recent interest in providing better quality medical services is booming. This has given rise to development of many new wireless technologies and has made Healthcare Sensor Network (HSN) as one of the popular areas of research. HSN is composed of a Wireless Body area Sensor Network (WBASN) and an Infrastructure Area Sensor Networks (IASN). In this paper, we discuss various challenges involved in designing and evaluating the performance of a HSN. As the sensor nodes are supported by batteries, they run on limited power supply. Thus, enhancing the network lifetime by efficient power management and effective energy harvesting strategies has become an important research goal. HSN operate in a highly dynamic environment and hence consume more power. Past research concentrated on energy harvesting using ambient sources such as solar, wind, thermal and vibration energy. Very little research has been done on harvesting thermal heat from the body and surrounding wasted heat. As there are many professionals like firefighters or first responders, who experience very high degree of activity and drastically increased physical and physiological operations in a short period of time, we explore the use of Small Thermoelectric Generators (TEGs) in harvesting energy from the body heat for such personal. We also discuss the potential application of harnessing surrounding waste thermal energy during a firefighting operation.
\end{abstract}

Index Terms-Energy harvesting, healthcare sensor networks (HSN), power management, thermoelectric generator (TEG), wireless body area sensor networks (WBASN).

\section{INTRODUCTION}

Recent global healthcare research has been focusing more on the quality of healthcare services. Providing affordable quality of healthcare to the consumers and reinforcing emerging technology of Healthcare Sensor Network (HSN) is of the paramount research interest. As defined in [1], HSN is a network of tiny monitoring devices, which may be mounted on the human being, embedded in the human body through invasive techniques, or may be integrated in the surrounding area. Network of wireless sensors when worn on the human body form a Wireless Body Area Sensor Network (WBASN), whereas, sensors embedded in the surrounding constitutes an Infrastructure Area Sensor Network (IASN) [1]. Both of them constitute a HSN which can be used in prognostic, diagnostic, and rehabilitative monitoring for numerous medical applications. Fig. 1 shows a generic WBASN architecture for the healthcare system. HSN can be used in order to monitor the physical parameters such, acceleration, pressure, muscle performance (EMG: Electromyogram) etc., and physiological

Manuscript received February 20, 2013; revised May 5, 2013

The authors are with School of Computing Sciences and Informatics, University of Cincinnati, Cincinnati, OH 45221-0008, USA (e-mail: jamtheas@mail.uc.edu,agrawadp@ucmail.uc.edu). parameters such as heart rate (ECG: Electrocardiogram), body temperature and blood pressure, etc. Some advanced monitoring systems monitor cardiac attack and fall detection of the elderly people and send feedback signals to the emergency medical services and/or doctors over the network. As a HSN allows real time data monitoring and analysis, a higher data sampling rate and transmission rate is desirable. For example, body temperature may need to be taken every 10 minutes; while other variables such as heart rate, blood pressure, blood ph may require a sampling rate of $20 \mathrm{~Hz}-100 \mathrm{~Hz}$ [1]. The processed data is then logged on to or transmitted to an attached actuator.

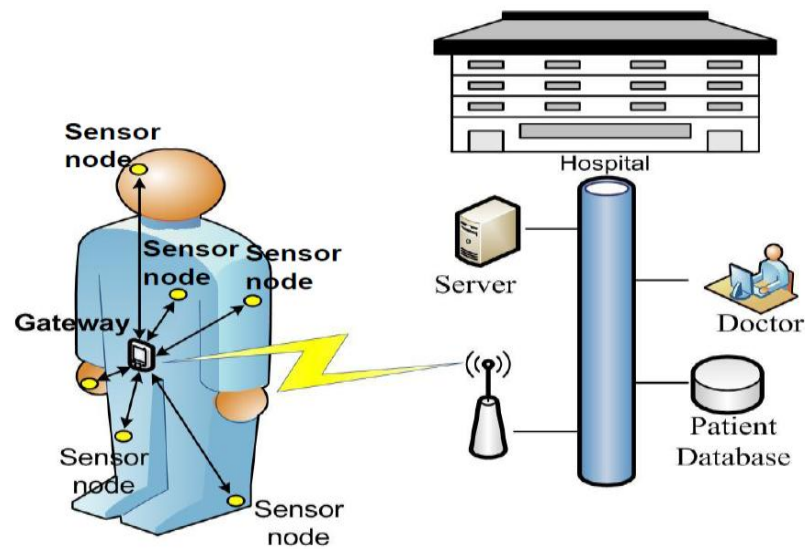

Fig. 1. Wireless body area network architecture in a medical healthcare system [1]

Several engineering challenges are involved in the design and performance of WBASNs which includes designing sensor nodes as light weight for easy portability and perform with accuracy, precision, and sensitivity and could last for a longer time. As each sensor nodes are powered by a battery, it is important to prolong the network lifetime using some energy harvesting and power management strategies. Energy conservation is a multi-objective problem dealing with different WSN parameters such as Quality of Service (QoS), transmission delays, etc. [1]. The need of WBASN to operate in a highly dynamic surrounding makes power management for data processing and transmission important. Protecting the patient's personal data from malicious users [2] also increases load on the power consumption. Fig. 2a shows the contents of a normal dummy packet sent from a senor node to the receiving station and Fig. $2 b$ shows the contents of the sensed packet after application of Elliptic Curve Cryptography techniques. This experimental data shows that packet sizes increases considerably on application of security measures, which leads to more energy consumption on transmission of packets. Thus application of security features increases the 
power consumption. Extensive research is being conducted to propose low power transmission protocols, efficient data processing on-chip algorithms, and energy harvesting techniques through ambient sources besides inclusion of light weight security mechanisms.

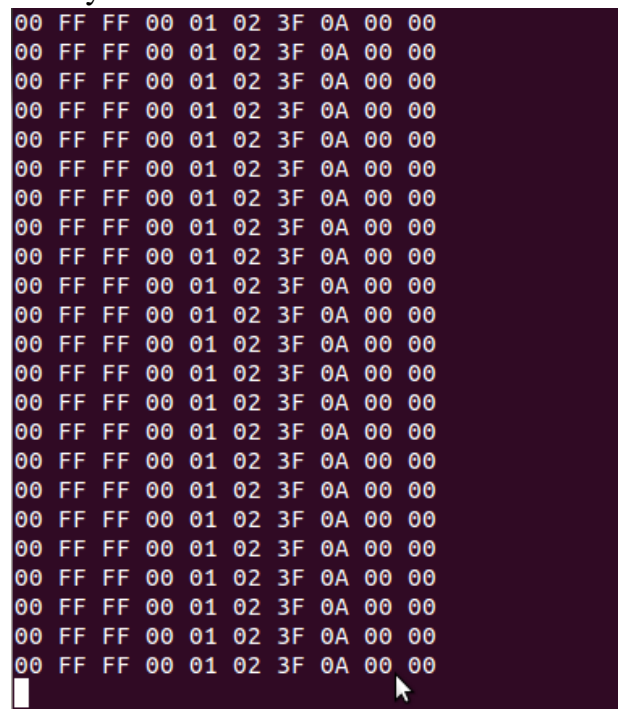

Fig. 2a. Normal dummy packet sent from a sensor

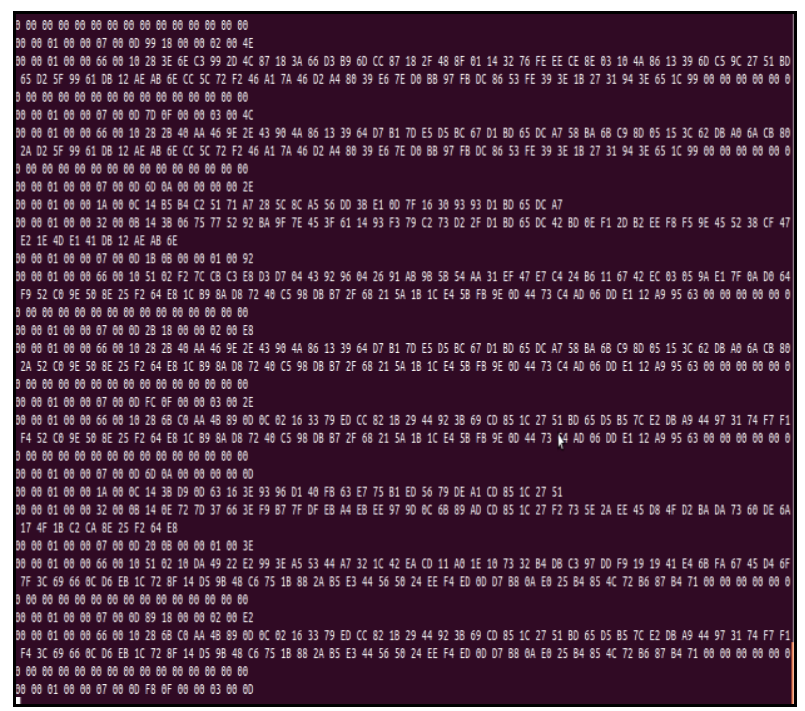

Fig. 2b. Packet size increases considerably on application of security measures

Node

Most of the wireless technologies are based on Radio Frequency propagation and includes IEEE 802.11.a/g/n (Wi-Fi), IEEE 802.15.1 (Bluetooth) and IEEE 802.15.4 (ZigBee). As the $\mathrm{Wi}-\mathrm{Fi}$ and Bluetooth were designed to ensure high data rates, they cannot ensure optimal power consumption [1]. ZigBee Alliance, recently stated that ZigBee HealthCare Standard can be used to monitor patients and elderly with a low power consumption mechanism. This protocol is optimized for applications with a low duty cycle, which need to spend most of its time in sleep mode [1]. Another transmission protocol that is being researched [1] uses human body as a communication channel.

Although there are some advantages of this method such as low power consumption and less RF interference, there are many limitations such as lower bandwidth and unknown health effects by the use of continuous electrical signals passing through the body. Current HSN architecture follows
RF protocols and consists of star topologies where sensed information is sent to the sink node, which aggregates and sends the data over the network for further analysis [1].

\section{BACKGROUND AND MOTIVATION}

\section{A. Power Management in WBASN}

WBASNs are powered by batteries which are limited in power. Thus, the user has to either frequently change the batteries or recharge them which is challenging for continuously monitoring and analyzing real time data in a highly dynamic environment. Thus, research is being done either to look for energy harvesting from an ambient source or use low power electronics and save power consumption, thereby somewhat prolonging lifetime of the network. We will briefly discuss both of these propositions. In a sensor node, power is consumed by each functional component such as transceiver, processing and sensing unit, data logger and microcontroller [3]. To reduce energy consumption of sensor nodes and prolonging network lifetime, a common method discussed in the literature is to adjust the duty cycle the sensor nodes. If a Crossbow sensor [4] operates at $1 \%$ duty cycle rather than full cycle, the power consumption is reduced significantly from $30 \mathrm{~mA}$ to $0.3 \mathrm{~mA}$ when the supply voltage is changed to $3 \mathrm{~V}$ [1].

\section{B. Wireless Sensor Network Protocol Stack}

Power management can be implemented in all the layers of WSN protocol stack. Table I indicates function of various layers in WSN protocol stack and energy conservation measures to be implemented in different protocol layers.

\begin{tabular}{|c|c|c|}
\hline Layer & Function & Power Management \\
\hline $\begin{array}{l}\text { Application } \\
\text { Layer }\end{array}$ & $\begin{array}{l}\text { Application } \\
\text { Software }\end{array}$ & $\begin{array}{l}\text { Focus on achieving power conservation } \\
\text { and } \\
\text { management. }\end{array}$ \\
\hline $\begin{array}{l}\text { Transport } \\
\text { Layer }\end{array}$ & \multicolumn{2}{|c|}{$\begin{array}{l}\text { Helps to maintain flow of } \\
\text { data }\end{array}$} \\
\hline $\begin{array}{l}\text { Network } \\
\text { Layer }\end{array}$ & $\begin{array}{l}\text { Routing data } \\
\text { by transport } \\
\text { layer } \\
\end{array}$ & $\begin{array}{l}\text { Efficient routing protocol } \\
\text { to save energy. }\end{array}$ \\
\hline $\begin{array}{l}\text { Data Link } \\
\text { Layer }\end{array}$ & $\begin{array}{l}\text { Minimize } \\
\text { collisions } \\
\text { between } \\
\text { neighbor } \\
\text { signals }\end{array}$ & $\begin{array}{l}\text { Power saving mode of } \\
\text { operations, wakeup and sleep schedules } \\
\text { implemented to } \\
\text { save power, radio turn off } \\
\text { during idle time slot. } \\
\end{array}$ \\
\hline $\begin{array}{l}\text { Physical } \\
\text { Layer }\end{array}$ & $\begin{array}{l}\text { Receive and } \\
\text { Transmit } \\
\text { data to/from } \\
\text { network } \\
\end{array}$ & $\begin{array}{l}\text { Design low power, tiny, low } \\
\text { cost transceiver, processing and } \\
\text { sensing unit. }\end{array}$ \\
\hline
\end{tabular}

\section{Energy Harvesting from Ambient Source}

Energy harvesting captures environmental energy such as solar, thermal, vibrations energy, RF energy, body heat, blood pressure etc., and harvests it so that WBASN can function almost indefinitely. This harvested energy is then converted to electrical energy to recharge the batteries for appropriate functioning of sensor nodes. There are several advantages of EH such as: Reducing the dependency on battery as BASN needs low power energy devices which can easily function on harvested energy from the ambient source, incur much smaller installation and maintenance costs and an effective long term 
solution for any WBASN.

\section{1) Solar EH systems}

For a volume of $1 \mathrm{~cm}^{2}, 100 \mathrm{~mW}$ of electrical energy can be harvested from a solar panel [5]. But, in an indoor environment, power drops drastically. Recently, efficient prototypes have been proposed for solar energy harvesting by Heliomote [6] and Prometheus [7]. Both of them are directly connected with energy storage devices. The basic principle behind the operation of a solar energy harvesting device is that it should be able to change the electrical operating point of a solar panel as per the incident light, so that Maximum Power Point is always maintained [1]. Some prototypes use a small photo sensor to sense the ambient light and force the solar panel not to exceed its Maximum power point [1]. Solar energy harvesting is a very realistic approach and a lot of research has been done to harvest energy and power the sensor senor for efficient functioning.

\section{2) Vibration EH systems}

Industries, bridges, and buildings experience mechanical vibrations which can be used to convert into electrical signals. Electromagnetic, electrostatic, and piezoelectric convertors translate mechanical motion into electricity which can be used to power WBASNs. In the past, powered wrist watch from vibration energy has been successfully implemented [1].

\section{3) RF Energy Harvesting systems}

Energy radiated by cell phone towers, wireless radio network, and TV towers can be harnessed to get DC voltage enough for powering sensor circuits. A power conversion circuit is used to convert the radio waves transmitted by the antennas into DC voltage. The generated energy can be stored in a capacitor and used to supply a constant flow of voltage to run the sensor device. Piezoelectric powered RFID system in shoes harvests energy from human walking or running have also been researched [1].

\section{4) Blood Pressure EH system}

For wearable sensors, powering energy can be harvested from human blood pressure. With the help of a special fabric, it might be possible to harness energy from blood pressure of a person wearing it. The resting heart rate is 60 beats $/ \mathrm{min}$ and heart stroke volume of $70 \mathrm{ml}$, for a normal human and about $0.93 \mathrm{~W}$ [7] power can be generated.

\section{5) Thermal energy harvesting system (TEH) from body heat and waste heat}

Thermal energy harvesting from waste heat in various industries is being researched extensively. Human body heat [8] can also be considered as a useful source for energizing low power wireless devices. We discuss about the body heat mechanics and its application in TEH in case of first responders in the following sections.

\section{METHODS AND EXPERIMENTS}

Human body heat and surrounding waste heat can also be considered as a useful source for energizing low power wireless devices such as wireless sensors used by the first responders and military officials. These personnel experience a larger degree of physical and psychological stress when either fighting fire or involved in battlefield. Research shows that most of the fatalities for firefighters occur on duty due to heart attack caused by heat stress [7]. It is required to constantly monitor their heart beat, blood pressure, and other vital parameters while they are in active operation. We can use ECG sensors which monitor heart rate and can send the data wirelessly to the monitoring station, which in turn sends alert if a firefighter shows symptoms of a heart attack by an unusual heart rate. This data can give information to their commanding chief if they need instant aid and can thus save their lives.

The commercial ECG sensors available run on battery and have a battery life of approximately 4 hours. As the data is sensed and sent to the monitoring station frequently, the battery will get drained off at a faster rate. This is due to the fact that the transmission of data consumes most of the battery power. We can use body heat and surrounding waste heat harvesting to power the low powered wearable sensors for continuous operation during fighting fire.

We discuss in brief the mechanics of Energy consumption in wireless sensor nodes. Energy spent for transmitting a $k$-bit message to distance $\mathrm{d}$ can be computed as below in equation 1 [3].

$$
E_{T x}(k, d)=E_{T x-e l e c}(k)+E_{T x-\text { amp }}(k, d)=E_{\text {elec }} \times k+k \times d^{2}
$$

where $E_{T x-e l e c}$ is the transmission electronics energy consumption, and $E_{T x \text {-amp }}$ is the transmitting amplifier energy consumption.

If we use data loggers instead of transmitting data, it will not allow real time monitoring. The data received will be useful only for later use. First responders experience high body temperatures while they are fighting fires. Research suggests that their body temperature can go high up to $104^{\circ} \mathrm{F}$. We will see how these elevated body temperature can be used to power the ECG sensors, worn by a person. We propose a thermal energy harvesting solution from body heat and surrounding hot air which can supplement the battery power of ECG sensors and can virtually make the system run for infinitely long time.

Core body temperature of a human being is more than his skin temperature. The temperature throughout the body varies according to external environmental conditions. Normal core body temperature is usually $98.6^{\circ} \mathrm{F}$ at atmospheric temperature between $70^{\circ} \mathrm{F}$ to $130^{\circ} \mathrm{F}$. Fig. 3 shows the change in body temperature of normal human being because of changes in the outside temperature. It indicates that at very high temperature body temperature tends to increases.

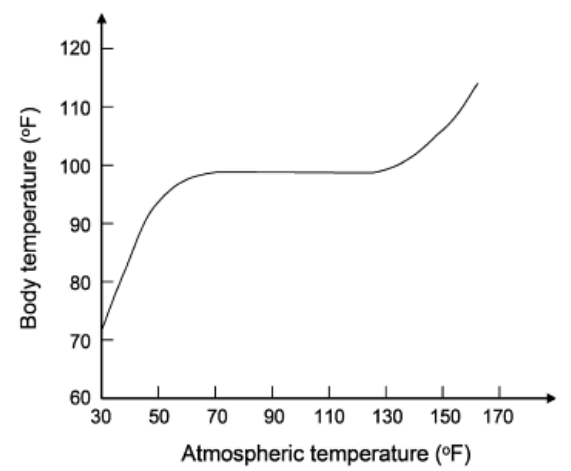

Fig. 3. Change in body temperature because of changes in atmospheric temperature [7] 
The amount of thermal energy dissipated also depends on the human activity. For example, during a sports activity, the person will have heat loss around $100 \mathrm{~W}$. Thus, for every $1^{\circ}$ rise in temperature, the heat dissipation increases by almost $13 \%$ [9]. Average specific heat of human body is $0.83 \mathrm{Kcal}$ which means that one degree change in temperature will occur when $0.83 \mathrm{Kcal}$ of body heat is changed [9].

According to first law of thermodynamics:

$$
Q=m C \Delta T
$$

where $Q$ is the amount of heat dissipated by mass m, having specific heat capacity $C$ when the change in temperature is $\Delta T$. We now model the heat flows from human body to air as follows in Fig. 4.

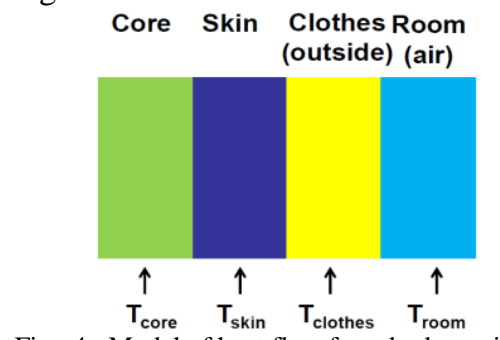

Fig. 4. Model of heat flow from body to air [9].

We apply this fact to an important theory of generating electrical energy from thermal energy. This is known as Seebeck Theory [10], which states that a difference in temperature in two dissimilar materials creates voltage [11]. Heat flow between adjacent regions can be given by [9]

$$
T_{1}-T_{2}=-I_{12} / A \times(d Q / d t)
$$

where $d Q / d t$ is the heat flow and $I_{12}$ is the insulation between two regions and $\mathrm{A}$ is cross sectional area.

In the process of calculating heat flow from a person's body to the surrounding area, we use the following temperature differences [9], where Ic is the insulation for clothing.

$$
T_{\text {skin }}-T_{\text {clothes }}=I c / A \times\left[(d Q / d t)_{r}+(d Q / d t)_{c}\right]
$$

For human clothing, the insulation provided per $\mathrm{cm}$ thickness is approximately $0.25^{\circ} \mathrm{C}-\mathrm{m}^{2} / \mathrm{W}-\mathrm{cm}$. It is supposed to be very high for firefighters clothing. So, if we consider change in temperature from $T_{\text {skin }}$ to $T_{\text {clothes }}$ for a firefighter, the difference will be positive as the body temperature will be larger than the clothing temperature, during active operations when their body temperature is elevated. This difference in temperature can generate voltage as per Seebeck's Theory. Similarly, the difference in temperature between firefighter's clothing and outside hot air (air in fire environment) can be provide us a high temperature difference which can be used to generate voltage [9].

$$
T_{\text {clothes }}-T_{\text {room }}=-I a / A\left[(d Q / d t]_{r}+(d Q / d t)_{c}+(d Q / d t)_{c}\right]
$$

According to Seebeck's theory, voltage generated is proportional to difference in temperature. Higher the temperature difference greater is the voltage generated.

$$
V=\alpha \times \Delta T
$$

where $\alpha$ is the Seebeck's coefficient. Seebeck coefficient for good thermoelectric material can range from $100 \mu \mathrm{V} / \mathrm{K}$ to

\section{$300 \mu \mathrm{V} / \mathrm{K}[10]$.}

We performed simulations and tried to verify our approach doing actual experiments. We measured the output voltage generated across thermoelectric materials with varying Seebeck coefficient randomly, ranging from $100 \mu \mathrm{V} / \mathrm{K}$ to $300 \mu \mathrm{V} / \mathrm{K}$, for different differences in temperatures. Fig. 5 shows the simulation results. It is observed that greater the Seebeck's coefficient and difference in temperature, higher is the amount of voltage generated. We then observed the actual output power generated depending on different values of load resistance and temperature difference across TEG. Fig. 6 shows that maximum power generated when the load varies and difference in temperature created across the two ends of thermoelectric generators is high.

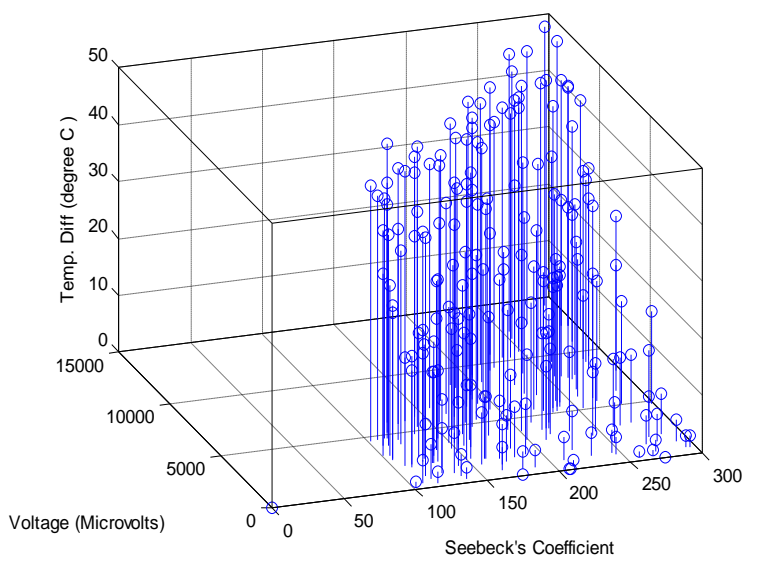

Fig. 5. Voltage in $\mu \mathrm{V}$ for different $\alpha$ and $\Delta \mathrm{T}$

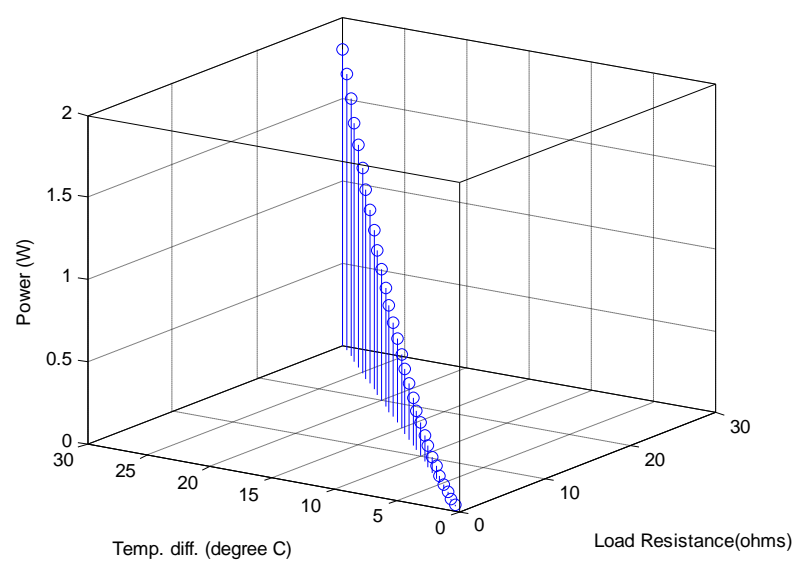

Fig. 6. Changes in output power depending on the value of Load Resistance and temperature difference across TEG

In our experimental setup, we used Peltier coolers TEC1-12710 [12], which act as thermoelectric generators, for converting body and surrounding heat energy into electricity. The flow of heat from hot end to cold end of Peltier cooler causes flow of electrons thereby generating voltage. It consists of many thermocouples of $n$ type and $p$ type semiconductor connected in series electrically and connected in parallel thermally [12]. We conducted experiments using TelosB sensor mote, which was sensing temperature every $250 \mathrm{~ms}$ and transmitting sensed data to the base station, located in the same premises. The data transmission rate is approximately $250 \mathrm{kbps}$. We observed for set of fully charged batteries in normal room conditions the batteries are exhausted within 6-7 hours of operation. We then connected in an integrated circuit a Peltier cooler along with TelosB sensor mote. The hot side of Peltier cooler was exposed to a 
hot air blower after every 5 minutes. This created a difference in temperature maximum up to $20^{\circ} \mathrm{C}$, varying in steps. The TelosB sensor was still sending data at $250 \mathrm{kbps}$. We observed that the hours of operation extended to 20 minutes as we connected 1 Peltier cooler to TelosB in a circuit. We can conclude that an optimal number of TEGs can extend the lifetime of sensor node by 2-4 hours of operation. This would be helpful in long firefighting operations or in defense applications where the warfighters do not get chance to recharge batteries and can lead to efficient monitoring.

But as having too many TEGs can lead to increase in cost of the monitoring circuit, we have to find an optimal tradeoff between cost and duration of operation of sensors in near future.

\section{CONCLUSION}

In this paper, we discussed the importance of healthcare sensor networks in today's medical health care services and also discussed various challenges involved in designing and enhancing the performance of hsn. we looked at different approaches of power management and energy harvesting through ambient sources in hsn. we also presented a theoretical and experimental approach for thermal energy harvesting from body heat and waste heat in the surrounding. we discussed how teg can be used to monitor firefighters and first responders where they experience elevated body temperature due to high degree of physical exertion and will also be exposed to very high surrounding temperatures. we discussed how peltier cooler, which act as teg can be integrated with sensor so as to prolong it duty of operation. we observed that for greater temperature difference more voltage will be generated. we observed that using a single peltier cooler, we are able to generate voltage of $0.25 \mathrm{mv}$ by surrounding waste heat and connecting 4 peltier coolers in series, it is possible to generate approximately $1 \mathrm{v}$. as the temperature difference will be very high for a typical firefighting operation, few peltier coolers can generate enough voltage to run the sensor circuitry.

There are wide range of applications of Thermal Energy Harvesting in determining cracks in airplane [13], studying concussion in players and detecting and alerting firefighters for the presence of harmful gases. In future we intend to study thermal energy harvesting technique for detection of harmful gases which the firefighters are exposed to during an actual active operation.

\section{ACKNOWLEDGMENT}

Our sincere thanks are due to Dr. Amit Bhattacharya from the Department of Environmental Health and Occupational Safety, University of Cincinnati, and Mr. Ashutosh Mani, Graduate Student from Department of Environmental Health and Occupational Safety for introducing us to this exciting application of Wireless Sensor Networks for firefighters. We would also like to thank our lab members for constant support and motivation.

\section{REFERENCES}

[1] D. Tze, H. Lai, R. Begg, and M. Palaniswami, "Health care sensor networks challenges toward practical implementation,"1st ed., Boca Raton, FL, CRC Press 2012, chapter. 01, pp. 2-5.
[2] M. Malik and D. P. Agrawal, "Secure Web Framework For Mobile Devices," GC'12 Workshop: The 4th IEEE International Workshop on Management of Emerging Networks and Services (GC'12 Workshop MENS 2012), Anheim, Ca, Dec. 3-7, 2012.

[3] C. Cordeiro and D. P. Agrawal, "Ad hoc \& Sensor Networks: Theory and Applications," World Scientific Publishing, Spring 2006, ISBN no. 81-256-681-3; 81-256-682-1, 2nd ed. 2011.

[4] Cross Bow Sensors Datasheet. [Online]. Available: http://bullseye.xbow.com:81/Products/Product_pdf_files/Wireless_pd f/TelosB_Datasheet.pdf

[5] K. Lin, J. Yu, J. Hsu, S. Zahedi, D. Lee, J. Friedman, A. Kansal, V. Raghunathan, and M. Srivastava, "Heliomote: enabling long-lived sensor networks through solar energy harvesting," SenSys '05 Proceedings of the 3rd international conference on Embedded networked sensor systems, 2005.

[6] R. O. Bonow, D. L. Mann, D. P. Zipes, and P. Libby, "Braunwald's Heart Disease: A Textbook of Cardiovascular Medicine," $9^{\text {th }}$ Edition, 2012.

[7] D. Smith, G. Horn, E. Goldstein, S. Petruzzello et al., "Firefighter fatalities and injuries, the role of heat stress and PPE," University of Illinois Fire Service Institute, Fire Fighter Life Safety Research Center.

[8] D. Hoang, Y. K. Tan, H. Chng, and S. Panda, "Thermal energy harvesting from human warmth for wireless body area network in medical healthcare system," Power Electronics and Drive Systems, 2009. PEDS 2009, International Conference, 2-5 Nov. 2009.

[9] P. Herman, "Physics of Human Body: A Physical view of Physiology," Springer Berlin Heidelberg New York 2007, ISBN no.978-3-540-29603-4, Third edition 2007.

[10] G. J. Synder, "Small thermoelectric generators," The Electrochemical Society Interface, Fall 2008, pp. 54-56.

[11] J. Randall and J. Jacot, "Is AM1.5 applicable in practice? Modelling eight photovoltaic materials with respect to light intensity and two spectra," Laboratoire de Production Microtechnique, IPR, STI, Ecole Polytechnique Fe'de'rale de Lausanne, CH-1015 Lausanne, Switzerland 2003.

[12] Peltier Cooler datasheet [Online]. Available http://www.hebeiltd.com.cn/peltier.datasheet/TEC1-12710.pdf

[13] D. Camphbell. (Oct. 2009). Energy Autonomous sensors find dents and cracks in airplanes. Available http://www.sciencedaily.com/releases/2009/10/091001095612.htm

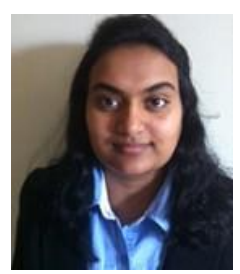

Anagha Jamthe was born in India. She is an IEEE student member since 2013. She received B.E degree in Computer Science Engineering from RTM Nagpur University India in 2006 and MEng. Degree with Computer Science major from University of Cincinnati, OH, USA in 2010. She is currently pursuing the $\mathrm{PhD}$. degree in School of Computing Sciences and Informatics (Computer Science Engineering), University of Cincinnati, OH, USA. Her current research interests include study of Wireless Body Area sensor networks and its various applications in Healthcare, Sports and Medicine.

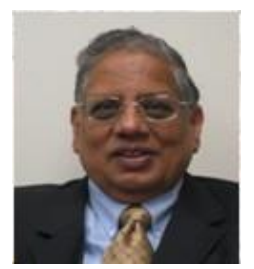

Dharma P. Agrawal has been the OBR distinguished professor at the University of Cincinnati, since August 1998. He is coauthor of textbooks Introduction to Wireless and Mobile System (3rd edition), and Ad hoc and Sensor Networks (2nd edition) and co-edited Encyclopedia on Ad Hoc and Ubiquitous Computing. He is a Fellow of the IEEE, ACM, AAAS, NAI, IACSIT, and WIF. He is a Golden Core member of the IEEE-CS and recipient of the IEEE Third Millennium Medal. He has published over 624 articles, 29 keynote speeches, 42 intensive courses, 6 patents and 25 invention disclosures, supervised 67 Ph.D. dissertations and led UCBT Bluetooth package. He has been on the editorial boards of IEEE Transactions on Computers, IEEE Computer, Journal of High Speed Computing, JPDC, and is serving IJCN, JECE, IJSIA, IJDSN, IJAHUC, IJAHSWN, JDSN, and IJWMC and founding EIC of the Central European Journal of Computer Science. His research interests include applications of sensor networks in monitoring Parkinson's disease patients and neurosis, applications of sensor networks in monitoring fitness of athletes' personnel wellness, applications of sensor networks in monitoring firefighters physical condition in action, efficient secured communication in Sensor networks, secured group communication in Vehicular Networks, use of Femto cells in LTE technology and interference issues, heterogeneous wireless networks, and resource allocation and security in mesh networks for 4G technology. 